\title{
A Pediatric Case of Thelaziasis in Korea
}

\author{
Chung Hyuk Yim¹, Jeong Hee Ko', Jung Hyun Lee', Yu Mi Choi', Won Wook Lee', Sang Ki Ahn², \\ Myoung Hee Ahn', Kyong Eun Choi' \\ Departments of ${ }^{1}$ Pediatrics and ${ }^{2}$ Ophthalmology, Gwangmyeong Sungae Hospital, Gwangmyeong 14241, Korea; ${ }^{3}$ Department of Environmental \\ Biology and Medical Parasitology, Hanyang University College of Medicine, Seoul 04763, Korea
}

\begin{abstract}
In the present study, we intended to report a clinical pediatric case of thelaziasis in Korea. In addition, we briefly reviewed the literature on pediatric cases of thelaziasis in Korea. In the present case, 3 whitish, thread-like eye-worms were detected in a 6-year-old-boy living in an urban area and contracted an ocular infection known as thelaziasis incidentally during ecological agritainment. This is the first report of pediatric thelaziasis in Seoul after 1995.
\end{abstract}

Key words: Thelazia callipaeda, thelaziasis, eye-worm, pediatric ocular parasite, ecological agritainment

\section{INTRODUCTION}

Thelazia callipaeda is an uncommon ocular parasite in Asia. The first human case was described in 1917 by Stuckey [1], and the first human infection in Korea was reported by Nakada in 1934 [2]. The recent large scale reports were presented by Sohn et al. [3] in Korea, and cases have been reported sporadically worldwide.

T. callipaeda is transmitted from animals to humans through the intermediate host, drosophilid flies [4]. Hence, the predisposing factors of thelaziasis have been determined as poor living conditions, low socioeconomic status, and poor hygiene, which are commonly seen in rural population [5]. However, its incidence has increased in urban areas also due to ecological learning and consumption of organic food. Therefore, thelaziasis is commonly found in urban areas as well as in rural areas. We report here a pediatric thelaziasis case with a brief literature review.

\section{CASE RECORD}

In September 2015, a 6-year-old boy was admitted to Gwangmyeong hospital due to fever, vomiting, abdominal pain, and eye pain in the left side. The child was managed by

\footnotetext{
- Received 24 January 2016, revised 20 May 2016, accepted 25 May 2016.

*Corresponding author (hygieia00@naver.com)

(C) 2016, Korean Society for Parasitology and Tropical Medicine

This is an Open Access article distributed under the terms of the Creative Commons Attribution Non-Commercial License (http://creativecommons.org/licenses/by-nc/4.0) which permits unrestricted non-commercial use, distribution, and reproduction in any medium, provided the original work is properly cited.
}

conservative treatment with intravenous cefotaxime $150 \mathrm{mg} /$ $\mathrm{kg} /$ day and levofloxacin eye drops after an ophthalmologic examination. The results of the blood test on admission were as

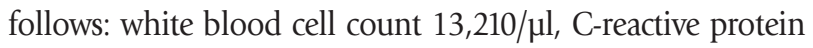
$7.191 \mathrm{mg} / \mathrm{dl}$, and eosinophil count $0 \%$. Parasitic, helminth eggs were not detected in stool examinations.

On the second day of admission, fever and abdominal pain were slightly improved, but the pain in his left eye was aggravated, and he was not able to open the left eye, in spite of eyedrop medication. After reconsultation with the ophthalmologist, 3 whitish, thread-like translucent worms were found in the left conjunctival sac. The ophthalmologist removed 3 moving worms from the left conjunctival fornix using intraocular fine forceps after administration of alcaine eyedrops, and fixed them in $10 \%$ formalin. Visual acuity of the child was within the normal range (right/left 0.9/0.8), and conjunctival injection was observed in the left eye.

The extracted worms were nematode adults and $8-16 \mathrm{~mm}$ in length. The vulva opening was located in the anterior region of the body, and the vagina opened anterior to the esophago-intestinal junction. The worms were identified as T. callipaeda at the Department of Parasitology, Hanyang University, Seoul, Korea. The worms were all females, and they were $8.0 \times 0.34$ $\mathrm{mm}, 15.0 \times 0.44 \mathrm{~mm}$, and $16.0 \times 0.45 \mathrm{~mm}$ in size. On light microscopic examinations, we observed a distinct buccal capsule at the anterior part of the worm, muscular esophagus, a lot of coiled larvae in the uterus, intestine, and a blunt straight posterior part with the anus and rectum. The striated solid cuticle of worms was also observed (Fig. 1).

The patient lived in an apartment beside a mountain at Gu- 

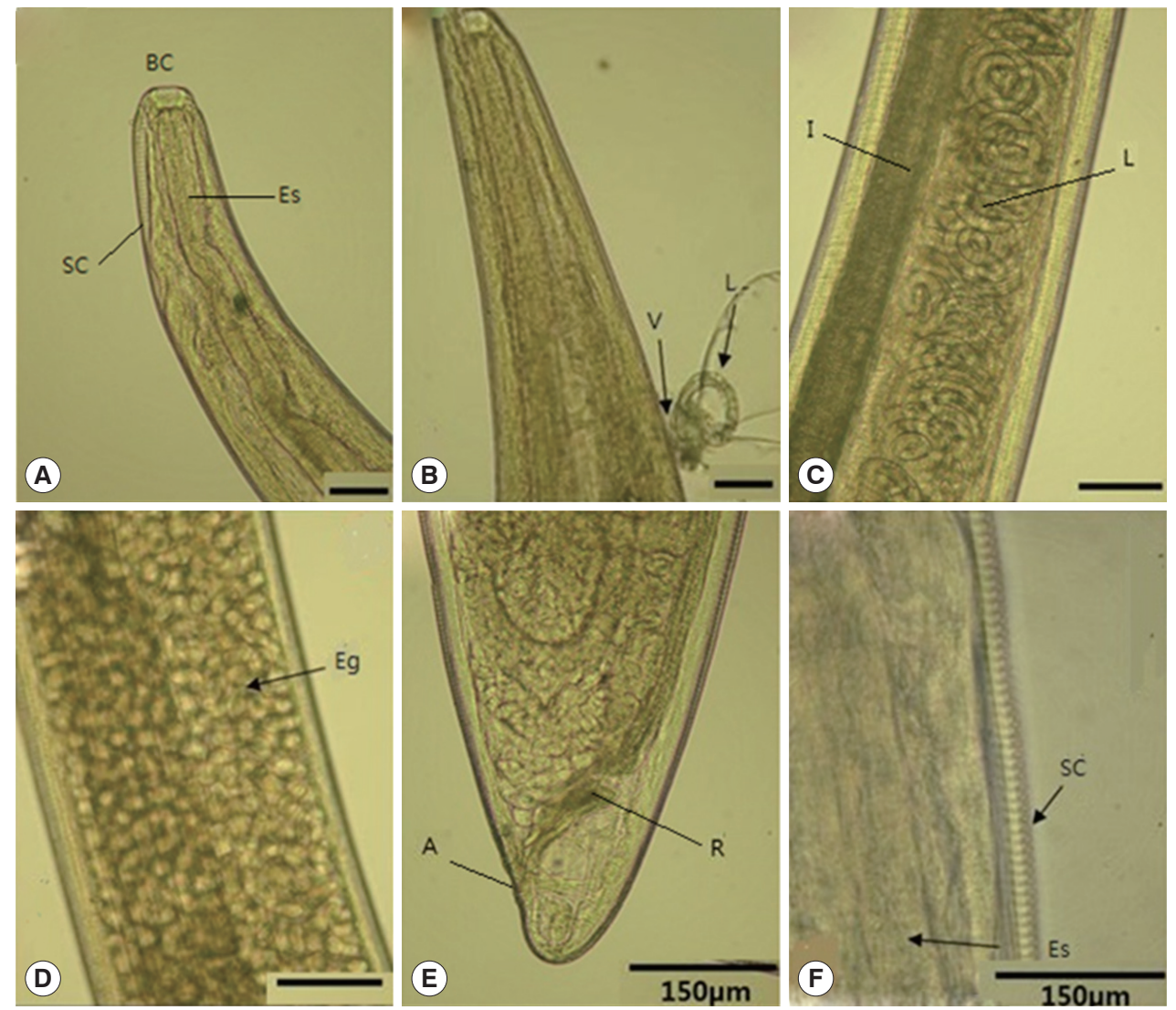

Fig. 1. Morphological features of Thelazia callipaeda (females) recovered in this case. (A, B) Anterior portion. BC, buccal cavity; SC, striated cuticle; Es, esophagus; V, vulva; L, larvae. Scale bar =150 $\mu \mathrm{m}$. (C, D) Middle portion showing the intestine (I), larvae (L), and eggs (E). Scale bar $=150 \mu \mathrm{m}$. (E) Caudal portion showing the rectum (R) and anus (A). (F) Esophageal level showing the esophagus (Es) and striated cuticle (SC).

Table 1. Reports of pediatric thelaziasis in Korea

\begin{tabular}{lccccc}
\hline Author & Patient age & Patient gender & Residential district & No. of worms & Year of publication \\
\hline Oh et al. [10] & 18 years & Female & Seoul & 2 & 1975 \\
Ryu et al. [11] & 15 years & Female & Seoul & 2 & 1986 \\
Kim et al. [12] & 9 years & Female & Seoul & 14 & 1990 \\
Hong et al. [6] & 7 months & Male & Uijongbu, Gyeonggi-do & 5 & 1995 \\
Oh et al. [13] & 6 months & Female & Yeongdeok-gun, Gyeonsangbuk-do & 3 & 2000 \\
Yim et al. [this study] & 6 years & Male & Seoul & 3 & 2016 \\
\hline
\end{tabular}

ro-gu, Seoul. He had attended kindergarten during the daytime, and did not own any pets. Lately, he had raised vegetables such as white radish and carrots at a garden, and had visited his grandmother who resided in the countryside, 2 weeks before admission. He also went on a picnic to an ecological park and had touched flowers and grass, 1 week before admission. He did not come in direct contact with animals and flies.

On the fourth day of admission, he was discharged. All clinical symptoms were improved at the time of discharge, and no worms were detected until 1 month follow-up.

\section{DISCUSSION}

We briefly reviewed the cases of pediatric thelaziasis in Seoul and Gyeonggi area since 1995 (Table 1). Most adults who had ocular thelaziasis visited the ophthalmologist due to conjunctival injection and foreign body sensation. However, most children who had ocular thelaziasis had a problem in describ- 
ing their clinical symptoms; hence, the diagnosis and management could be more delayed if the patient is younger.

In our case, the patient did not live in a rural area, but his outdoor activity might be a significant course of infection. All cases of thelaziasis in children living in Seoul and Gyeonggido were reported before 1995 [6], and this indicates that urbanization lowered the incidence of human thelaziasis. This case has a special significance with respect to the area of residence and the young age.

Removal of worms is the mainstay of management and diagnosis of ocular thelaziasis. Additional management options such as medication and surgery are not necessary. T. callipaeda commonly affects the anterior segment of the eye, and it can also cause severe damage to the posterior segment [7], and even retinal detachment [8]. Conjunctival and corneal injuries, traumatic conjunctivitis facilitate the introduction of the larvae into the subconjunctival space and vitreous cavity [5]. Therefore, complete elimination of worms and careful ophthalmologic follow-up examination are required in all patients who have experienced ocular thelaziasis.

Prevention of ocular thelaziasis includes avoiding flies in summer that are attracted due to excessive sweating or shedding tears. The methods to avoid flies include bed nets at night, keeping the face clean during summer, among people living in the rural area as well as in the urban area. Also, an early ophthalmologic examination is required in the presence of eye pain to detect ocular thelaziasis.

The infection rate is high in preschool children, elders, and farmers living in China [9]. However, the reported pediatric cases of thelaziasis in Korea showed no significant predominance for preschool age. Table 1 shows the pediatric data of ocular thelaziasis in Korea. The number of reports is 6 papers, and the residential districts are Seoul/Gyeonggi (5) and Yeongdeokgun (1).

In this study, the authors incidentally discovered an ocular thelaziasis case in a 6-year-old boy living in an urban area and who had eye pain with viral febrile illness. This report describes the first case of pediatric thelaziasis since 1995 in Korea. He might have been infected during ecological agritain- ment in rural areas. We should pay more attention to prevention of thelaziasis since children in the kindergarten are becoming actively involved in activities outside the farm. Physicians should always keep in mind that ocular pain could be due to parasites or as a result of some systemic disease in spite of maintaining good hygiene.

\section{CONFLICT OF INTEREST}

We have no conflict of interest related to this work.

\section{REFERENCES}

1. Stuckey E. Circumocular filariasis. British J Ophthalmol 1917; 1: 542.

2. Nakada K. Examples of the parasite Thelazia callipaeda in humans in Korea. J Chosen Med Assoc 1934; 24: 939-944.

3. Sohn WM, Na BK, Yoo JM. Two cases of human thelaziasis and brief review of Korean cases. Korean J Parasitol 2011; 49: 265-271

4. Otranto D, Eberhard ML. Zoonotic helminths affecting the human eye. Parasit Vectors 2011; 23: 41.

5. Krishnachary PS, Shankarappa VG, Rajarathnam R, Shanthappa M. Human ocular thelaziasis in Karnataka. Indian J Ophthalmol 2014; 62: 822

6. Hong ST, Park YK, Lee SK, Yoo JH, Kim AS, Chung YH, Hong SJ. Two human cases of Thelazia callipaeda infection in Korea. Korean J Parasitol 1995; 33: 139-144.

7. Krishnacharya PS, Shankarappa VG, Rajarathnam R, Shanthappa M. Human ocular thelaziasis: a case report from Karnataka. Indian J Res Rep Med Sci 2011; 1: 38-46

8. Chen W, Zheng J, Hou P, Li L, Hu Y. A case of intraocular thelaziasis with rhegmatogenous retinal detachment. Clin Exper Optometry 2010; 93: 360-362.

9. Zheng DF, Xiao N, Chen XW, Ai Z, Tian HC, Qing Z, Li DY, Chen JP, Liao L. Distribution and harmfulness of human thelaziasis in mainland of China. 2010; 37: 32-38.

10. Oh CK, Youn WS, Cho SY, Seo BS. A case report of human thelaziasis. J Korean Ophthalmol Soc 1975; 16: 135-138

11. Ryu JS, Im KI, Byun YJ, Kim SC. A case of human thelaziasis in Korea. Korean J Parasitol 1986; 25: 83-84.

12. Kim DC, Shin H. Two cases of human thelaziasis. J Korean Ophthlalmol Soc 1990; 31: 801-805 (in Korean).

13. Oh TS, Ahn Y. A case of Thelazia callipaeda conjunctival infestation. J Korean Ophthalmol Soc 2000; 41: 1437-1440 (in Korean). 
